\title{
Paradigm-shift: radiological changes in the asymptomatic iNPH-patient to be: an observational study
}

\author{
D. C. Engel ${ }^{1^{*}} \mathbb{D}$, S. D. Adib ${ }^{1}$, M. U. Schuhmann ${ }^{1}$ and C. Brendle ${ }^{2}$
}

\begin{abstract}
Background: Many radiological signs are known for the diagnosis of idiopathic normal pressure hydrocephalus (iNPH). However, there is little information about these signs in the pre-symptomatic phase. For pathophysiological investigative purposes we conducted a descriptive image analysis study on pre-symptomatic patients.

Methods: Patients that had contact with either the neurological or neurosurgical department of the university hospital Tuebingen from 2010 through 2016 with magnetic resonance images $>3$ years before onset of symptoms, were included. The date of onset and severity of symptoms, date of first imaging and birth date were recorded. Evan's index (EI), width of the third ventricle (3VW), tight high convexity (THC), Sylvian fissure, extent of white matter hyperintensities and aqueductal flow were assessed in images before and around symptom onset.

Results: Ten patients were included. In all ten patients the first symptom was gait disturbance. Nine of ten pre-symptomatic images showed classic signs for iNPH. El showed a significant increase between the pre-symptomatic and symptomatic phase. 3VW showed a trend for increase without significance. THC changed back and forth over time within some patients.

Conclusions: In accordance with the scarce literature available, radiological changes are present at least 3 years before onset of iNPH-symptoms. El seems to be a robust measure for pre-symptomatic radiological changes. Extrapolating the data, the development of iNPH typical changes might be an insidious process and the development of THC might be a variable and non-linear process. Further studies with larger sample sizes are necessary to put these findings into the pathophysiological perspective for the development of iNPH.
\end{abstract}

Keywords: Etiology, Pathophysiology, Idiopathic normal pressure hydrocephalus, Tight high convexity, Ventriculomegaly, Evan's index

\section{Background}

The etiology of idiopathic normal pressure hydrocephalus (iNPH) has been a research focus since its first description in 1965 [1]. The overall prevalence is estimated to be 10.2-31.4/100,000, and might even be 5900/100,000 in the $>80$ year olds [2-4]. Better understanding of the pathophysiology and better treatment possibilities are needed for our aging populations.

\footnotetext{
*Correspondence: doortje.engel@med.uni-tuebingen.de

1 Department of Neurosurgery, University Hospital of Tuebingen,

Hoppe-Seyler-Strasse 3, 72076 Tuebingen, Germany

Full list of author information is available at the end of the article
}

The pathophysiology of iNPH is not completely understood currently. Studies indicate that a prolonged, possibly lifelong process underlies the development of what we know as iNPH [5-7]. Known changes in pathophysiological parameters measured at the time of symptomatic iNPH include decreased subcortical cerebral blood flow (CBF) [8], decreased flow in the internal carotid artery [9], reduced flow velocity in the superior sagittal sinus [10], a higher incidence of retrograde jugular flow during the Valsalva maneuver [11], increased aqueductal cerebrospinal fluid (CSF) flow velocity [12-14], decreased cervical CSF stroke volume [9], decreased elastance of the intradural compartment and increased CSF pulsatility 
and outflow resistance $[15,16]$, increased lactate concentrations in the lateral ventricles [17], deep and (partially reversible) periventricular white matter hyperintensities (WMH) [18-20] and increased neurofilament light chain protein in CSF as a sign of neurodegeneration [21,22].

The hydraulic press hypothesis of Hakim and Adams explains the development of iNPH by disturbance of CSF-resorption leading to an increased outflow resistance, which in turn leads to an initial increase of ICP. The authors postulate that due to the increased ICP the ventriculomegaly occurs from the indirect linearity of pressure and surface at constant pressure according to Pascal's Law [23]. However, it lacks an explanation for the asymptomatic phase of increased ICP. Furthermore, a transmantle pulsatile gradient within the CSF of the cerebral ventricles in symptomatic iNPH patients could not be proven [24].

Another theory exists called the two-hit hypothesis, comprised of the occurrence of a benign external hydrocephalus during infancy followed by ischaemic insults leading to deep and periventricular WMH [7]. The first hit results in a larger intracranial volume in $\mathrm{iNPH}$ patients [7]. The second hit, ischemic insults, could be induced by worsening of periventricular ischemia [25, 26], softening of the brain [27], or decreased CSF resorption [28, 29] leading to the beginning of symptoms [7].

A third, hydrodynamic, hypothesis of Greitz is explained as "restricted arterial pulsation hydrocephalus" or "increased capillary pulsation hydrocephalus" [30]. An increasing rigidity of the cerebral arteries by arteriosclerosis could lead to the inability to attenuate the pulsations of CBF into the brain parenchyma, causing a reduction of the periventricular metabolism and degeneration of the periventricular parenchyma [30-32].

In addition to the above-mentioned mechanical hypotheses, genetic predispositions such as homozygous apolipoprotein E allele on chromosome 19, play an important role in familial iNPH [33-35].

The above-mentioned pathophysiological parameters cannot prove any of the stated hypotheses, because all data have been logically derived in patients who already had symptomatic iNPH. Therefore we aimed to investigate the presence of iNPH-typical changes in diagnostic images in the pre-symptomatic phase of iNPH.

\section{Methods}

\section{Patient inclusion}

The diagnosis of iNPH was screened as either probable or possible and secured according to international guidelines by an objective improvement of gait in the $10 \mathrm{~m}$ walking test after either (repetitive) CSF tap-test(s) or lumbar drainage for 3 days [36-39]. Those patients with imaging available 3 or more years before symptom-onset, that had contact with the neurological or neurosurgical department of the university hospital Tuebingen for the diagnosis of iNPH from 2010 thru 2016 were included. Time of symptom-onset was extracted from the medical history. The 3-year cut-off was chosen because of the insidious onset of iNPH. Patients with an unclear hydrocephalus-type, possibly due to neurotrauma, meningitis or intracranial bleeding or with an unclear symptomonset or origin, i.e. due to simultaneous high-grade cervical spinal stenosis were excluded. Age, time of symptom onset, time of first imaging and first presenting symptoms were assessed. All of the imaging data (MRI and $\mathrm{CT}$ ) were included in this retrospective analysis.

\section{Image analysis}

Imaging data were acquired on CT and MRI scanners approved for clinical routine use. Data were reconstructed according to clinical standard orientated at the corpus callosum in the axial slices and at the brain stem in the coronal slices. For image analysis in CT, the axial slices and where available the coronal slices were assessed. For image analysis in MRI, the axial fluid attenuated inversion recovery (FLAIR) sequence and where available the axial, coronal or sagittal T2-weighted images were assessed. Images after treatment by ventriculoperitoneal shunt implantation (VPS) were not included.

A neuroradiologist $(\mathrm{CB})$ scored the imaging data being blinded for the hypotheses of this project and the clinical data. The following measurements were obtained:

1. Evan's index (EI) was determined as the ratio of the maximum transverse diameter of the frontal horns of the lateral ventricles and the diameter of the inner skull [40].

2. The width of the third ventricle (3VW) was measured in the axial plane in the mid-portion of the third ventricle.

3. Tight high convexity (THC) of the subarachnoid space was measured following the method of Narita et al. [41] in both transverse and coronal sections and rated as: 0 , normal; 1 , mild tightness; 2 , moderate tightness; 3 , severe tightness.

4. Width of the Sylvian fissure was assessed only on transverse sections as: 0 , narrowed, 1 , normal; 2 , mildly dilated; 3 , severely dilated [41].

5. Periventricular and deep WMH were scored according to Fazekas [42].

6. The existence of aqueductal flow voids/jets was documented if appropriate MR-sequences were available.

The callosal angle was not included due to missing coronal sections in many of examinations. 


\section{Statistics}

Normal distribution of the data was denied by the Shapiro-Wilk test. Wilcoxon text was performed to compare EI and 3VW before and after onset of symptoms in patients with available imaging data at both time points.

\section{Results}

From 2010 through 2016, 382 patients suspected of having NPH had contact with either the neurological or neurosurgical department. Ten patients met inclusion criteria and were finally included in the study with a male-to-female ratio of 1:1 and a mean age at symptomonset of 77.4 years (range 70.9-88.7).

\section{Clinical data}

Seven patients were treated by VPS and improved longterm ( $>1$ year); the other 3 either refused treatment or received repetitive lumbar punctures. Gait disturbance was the first reported symptom in all cases. Nine patients suffered all three symptoms of the Hakim triad at the time of diagnosis. Only one patient reported no cognitive deficits and had a Mini Mental State Examination (MMSE) score at time of diagnosis 30/30. The other patients did indicate cognitive deficits. In this group MMSE ranged from 12 to 29/30. Nine patients were diagnosed with hypertension before symptom-onset.

\section{Imaging data}

On average the first imaging examination (CT or MRI) was conducted -6.2 years to symptom onset (range 3.1-10.3). In 2 cases 3 MRIs were available at $-6.1,-2.8$ and 0.2 and $-10.3,-4.1$ and 0.5 years to symptom onset respectively. All time points are relative to symptom onset. Details of the available imaging data are shown in
Table 1. An illustrative case (patient 7) with increasing radiological changes from the pre-symptomatic to symptomatic phase is shown in Fig. 1.

\section{Evan's index and width of 3rd ventricle}

EI was $\geq 0.3$ in all cases at all imaging times. Figure 2 shows EI over time. The values most prior to and closest to symptom onset were included. The same was done for 3VW (see Fig. 3). One patient suffered from bithalamic ischemia 4 years before symptom onset. This patient was not excluded because EI and 3VW had already been enlarged before the occurrence of the ischemic event. Six patients had available imaging data at time points before and after onset of symptoms. In the remaining four patients, the second examination was performed shortly before symptom onset. In these six patients, the mean value of EI was significantly higher after symptom onset than at the pre-symptomatic time point (mean value $0.39 \pm 0.02$ and $0.35 \pm 0.03$, respectively, $\mathrm{p}=0.0354$ ) 3VW did not show a significant difference between both time points (mean value $18.3 \pm 3.0$ and $15.8 \pm 3.4$, respectively).

\section{Tight high convexity}

Data on THC were available on the first image in nine patients. Seven of nine had a positive THC-score of 1,2 or 3 . The 2 patients who had no THC score of 0 in the first image at -10 and -6 years, did have mild score of 1 at -4 years or a moderate score of 2 , at -1 year on the second available image. Two patients had mild THC of score 1 before symptom onset at -5 and -7 years, scored 0 after symptom onset at +1.5 and 0 years. In the remaining 5 patients, THC score increased over time. All patients are depicted in Fig. 4.

Table 1 Images available per patient

\begin{tabular}{|c|c|c|c|c|c|c|c|c|}
\hline \multirow[t]{2}{*}{ Patnr } & \multicolumn{2}{|l|}{ Imaging 1} & \multicolumn{2}{|l|}{ Imaging 2} & \multicolumn{2}{|l|}{ Imaging 3} & \multicolumn{2}{|l|}{ Imaging 4} \\
\hline & Timepoint & Modality & Timepoint & Modality & Timepoint & Modality & Timepoint & Modality \\
\hline 1 & -4.7 & $\mathrm{CT}$ & -3.3 & $\mathrm{CT}$ & -0.9 & MRI & 2.1 & $\mathrm{CT}$ \\
\hline 2 & -5.4 & $\mathrm{CT}$ & -0.1 & $\mathrm{CT}$ & 0.2 & $M R I$ & & \\
\hline 3 & -6.1 & MRI & -2.8 & $M R I$ & 0.2 & $\mathrm{CT}$ & 1.9 & $\mathrm{CT}$ \\
\hline 4 & -3.1 & $\mathrm{CT}$ & 0.9 & $\mathrm{CT}$ & & & & \\
\hline 5 & -3.6 & $\mathrm{CT}$ & -0.1 & MRI & & & & \\
\hline 6 & -7.8 & MRI & 1.5 & MRI & 1.9 & $\mathrm{CT}$ & & \\
\hline 7 & -10.3 & MRI & -4.1 & MRI & -1.4 & $\mathrm{CT}$ & 0.5 & MRI \\
\hline 8 & -6.1 & MRI & 0.7 & $M R I$ & 2.0 & $\mathrm{CT}$ & & \\
\hline 9 & -9.2 & $\mathrm{CT}$ & 0.8 & MRI & & & & \\
\hline 10 & -5.8 & $\mathrm{CT}$ & 2.0 & MRI & & & & \\
\hline
\end{tabular}

Available imaging according to time-to-symptom-onset including first image available around symptom-onset, either CT or MRI. Patnr: patient number; timepoint: interval between imaging and onset of symptoms in years 


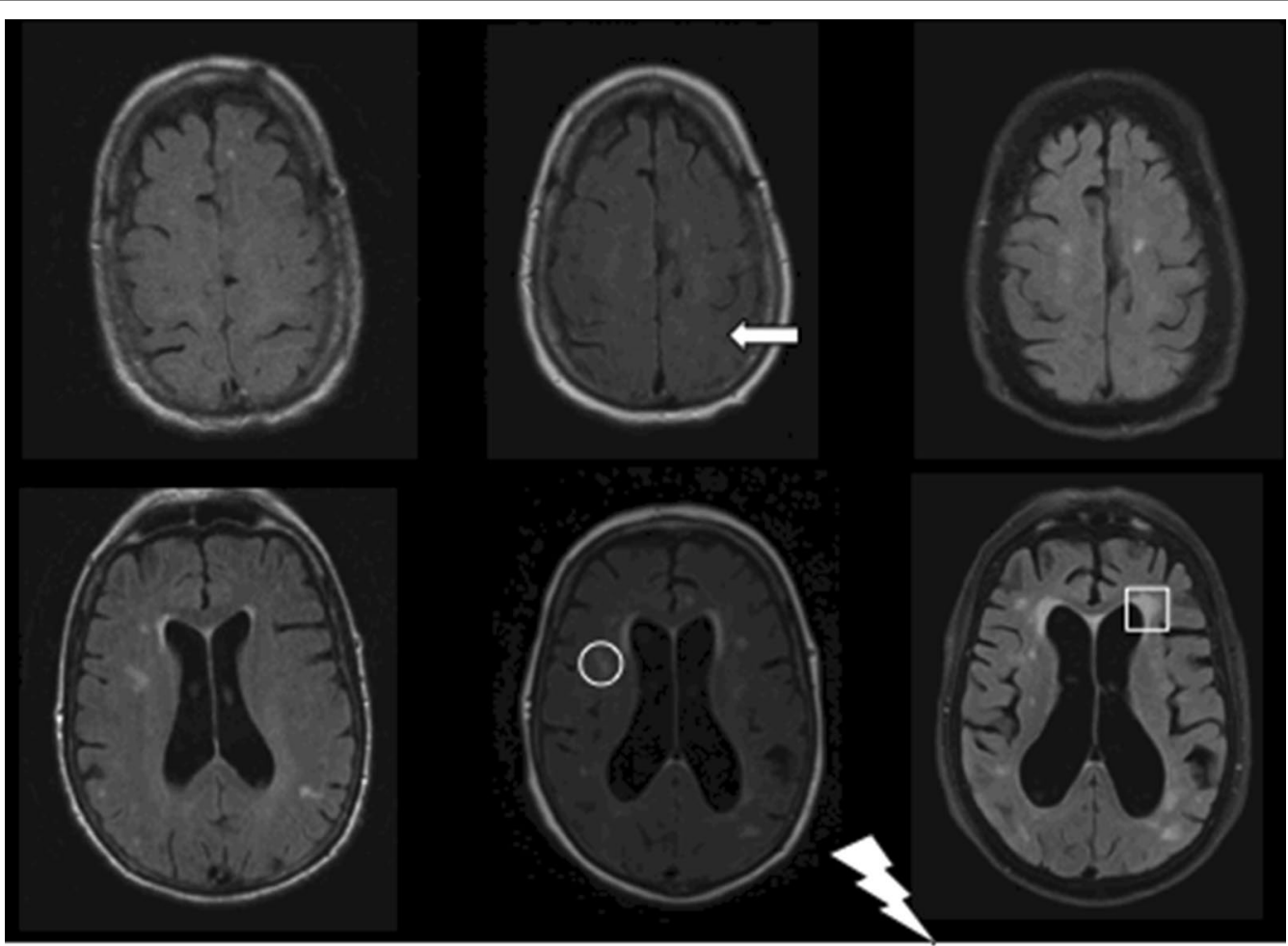

\section{First symptom at $83 y$}

Fig. 1 Pre- and post-symptomatic magnetic resonance images of a patient that developed symptoms after 10 years. The MRI images show the changes in ventricle width, deterioration of white matter hyperintensities (circle: deep; square: periventricular) and tight high convexity (arrow) all before onset of symptoms (thunderbolt) of a typical case. Left: 10 years before symptom onset. Middle: 4 years before symptom onset. Right: 6 months after symptom onset

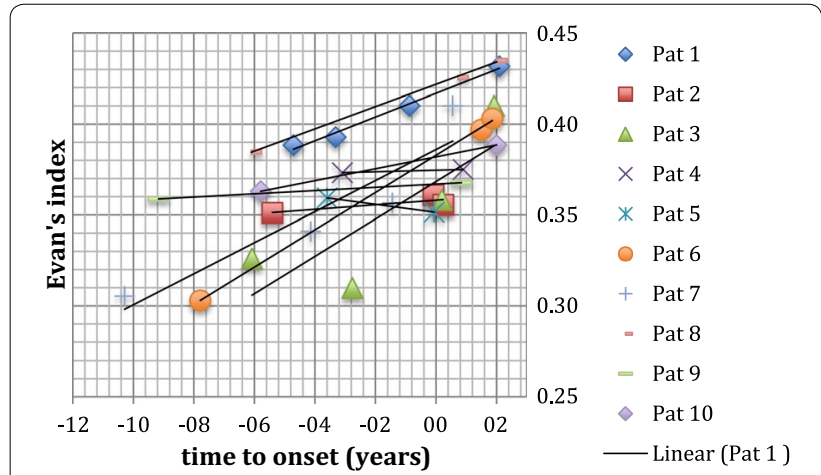

Fig. 2 Change in Evan's Index over time. Each patient is depicted separately. El was above 0.3 in all patients and increased over time significantly. Time 0 is time of symptom onset

\section{Other imaging findings}

All patients with the relevant MRI $(n=7)$ showed periventricular and deep WMH. Only in 4 patients was MRI-data available at least 3 years before symptom-onset. The Fazekas score for both deep and

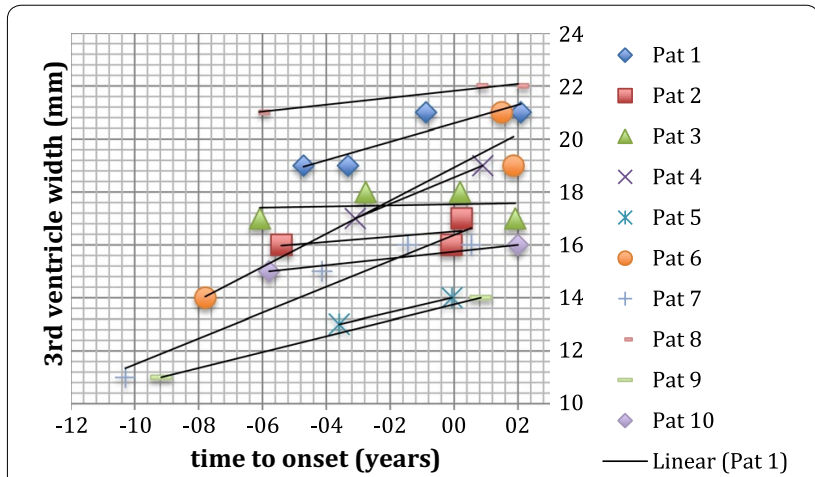

Fig. 3 Change in third ventricle width over time. Each patient is depicted separately. Third ventricle width (in $\mathrm{mm}$ ) was above $9 \mathrm{~mm}$ in all patients and increased over time. Time 0 is time of symptom onset

periventricular WMH ranged from mild to severe (score 1-3) [42]. Aqueductal flow voids could be determined in 7 patients with T2-weighted sagittal MRI images and was present in all 7 cases. No major Sylvian fissure atrophy was seen. 


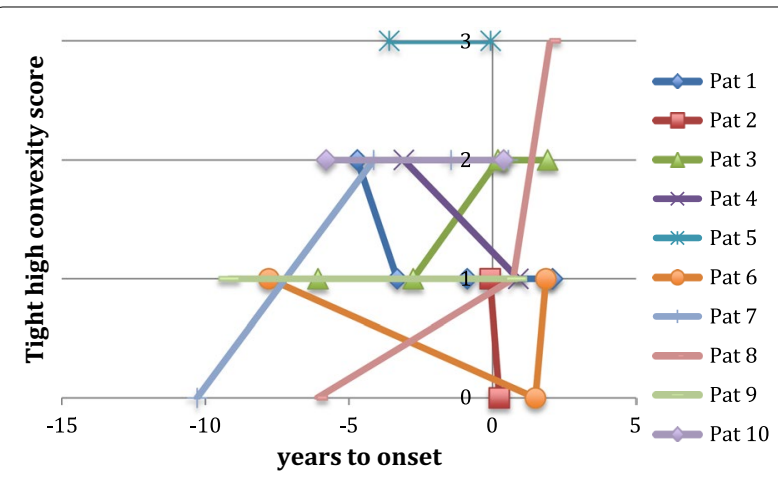

Fig. 4 Change in tight high convexity over time. Each patient is depicted separately. THC varied through time within patients. Time 0 is time of symptom onset

\section{Discussion}

This study shows that increased ventricular width, aqueductal flow void, deep and periventricular WMH and fluctuating THC precede symptom-onset of iNPH by at least 3 years. The presence or absence of THC seems to be a dynamic process varying in a non-linear fashion. This gives new insights into the pathogenesis of iNPH.

EI seems to be a robust measure for pre-symptomatic radiological changes in iNPH. EI increased significantly from the pre-symptomatic images to the time point of iNPH diagnosis. Extrapolation of the obtained data, assuming that EI change is linear over time, implies that the pathophysiological start of iNPH could be around 10-15 years before symptom onset. An on-going change in ventricular width might contradict the two-hit hypothesis on iNPH pathogenesis of the presence of benign external hydrocephalus in infancy followed by deep white matter ischemia later on as proposed by Bradley et al. [7, 43]. However, no proof of either theory exists to date. Further pre-symptomatic and long-term investigations are necessary. Moreover, it is conceivable that different subtypes of iNPH exist with different pathophysiological pathways.

Unlike Iseki et al. [5], our data show ventriculomegaly in almost all patients with only mild to moderate THC. A selection bias logically exists in the current study due to the retrospective nature and its inclusion criteria. The imaging studies at the pre-symptomatic time points were performed because of different neurological indications not connected to iNPH, whereas Iseki et al. have investigated a population-based cohort. Nevertheless, our data show a certain dynamic trend of THC in both increasing and decreasing throughout the years. These data suggest rather a variable than linear development of THC over time, possibly due to build up of internal CSF pressure followed by pressure decline after widening of the ventricles due to cell loss. Although the hypothesis of a transmantle gradient in pulsatile ICP in the lateral ventricles was disproven in 2010 [44], pressure was measured only in symptomatic patients. As CSF pulsatility index and CSF outflow resistance are increased in iNPH patients in the symptomatic phase $[15,16]$, increased ICP in the pre-symptomatic phase cannot be ruled out completely.

The presence of deep WMH together with the high prevalence of hypertension in our patients supports aspects of the hydrodynamic hypothesis of Greitz [45]. Approximately $50-60 \%$ of the $70-79$ year old general population had hypertension according to a health survey 1988-1991 [46]. A more recent German survey showed that $60 \%$ of $50-59$ year olds and $75 \%$ of $70-79$ year olds suffer from hypertension [47]. Also signs of cerebrovascular disease were found in post-mortem and biopsy studies in iNPH patients [48-50]. Next to increasing blood pressure with age, there is a reduction of the dampening effect in cerebral arteries during aging due to stiffening of the arteries. This would further support the hydrodynamic theory [51]. In contrast, Qvarlander et al. did not find a significant difference in total cerebral artery inflow pulsatility index between healthy and iNPH patients [9]. It is thus unclear, if the extracranial arterial pulsatility reflects intracranial pulsatility, since the extracranial compartment is highly compliant as opposed to the intracranial compartment.

Transependymal edema or microangiopathic ischemia presenting as periventricular WMH was seen in all of our patients and existed in at least four patients before symptom onset. Previous data show that acetazolamide can reduce periventricular WMH with or without corresponding gait improvement in some but not all patients [19]. Acetazolamide inhibits CSF production in the choroid plexus, inhibits aquaporin-mediated water transport and normally leads to a CBF increase by vasodilation [52]. iNPH patients have an impaired cerebrovascular reactivity as shown before $[53,54]$. The cerebrovascular reactivity was improved by acetazolamide in patients that showed clinical improvement after shunt surgery but not in the so-called non-responders [54]. All of our patients showed improved gait after shunt surgery or multiple lumbar punctures, while having had periventricular WMH for several years. Even years after symptom onset patients can improve after shunt surgery [55]. It remains unclear when the point of no return kicks in, where transependymal edema turns into ischemic tissue. To date no asymptomatic patient has received therapy for dilated lateral ventricles with typical iNPH imaging signs, as surgery has risks. Our data suggest that due to long-term progression a follow up of these asymptomatic patients with ventriculomegaly is necessary. These patients should 
be informed about the risk of developing iNPH symptoms in order to offer treatment at an early stage and preserve quality of life for as long as possible.

Limitations of this study include the retrospective nature of the investigation as well as the non-standardized documentation of clinical data, partially due to the fact that the patients were examined in two different departments. Furthermore, only low numbers of patients could be included due to the cut-off of available imaging at 3 years before symptom onset. The 3-year cutoff was set arbitrarily. Many patients already had symptoms 1-2 years before seeking medical advice. As symptoms start insidiously, imaging conducted at least 3 years before implied symptom-onset was considered to be realistically pre-symptomatic. The available imaging data was inconsistent, in some cases only CTs were available and not all parameters could be derived. This might have introduced biased assessments of the imaging data. However, length measurements as used in the EI and width of the third ventricle should be comparable between both modalities, and other measurements were based on rough scoring systems. Confounding is the fact that only iNPH-patients were investigated and not a populationdwelling cohort. However, it is likely that these changes are linked to iNPH, as patients in a previous study all developed iNPH-symptoms and very few asymptomatic participants were found after 10 years of scanning [5]. Due to the limited patient number, the statistical analysis can only give a preliminary overview. More longitudinal prospective research needs to be conducted similar to the work of Iseki et al. combining large cohort MRI data with clinical and neuropsychological data [5].

\section{Conclusions}

In accordance with scarcely available literature radiological changes are present at least 3 years before onset of iNPH-symptoms. Evan's index seems to be a robust measure for pre-symptomatic radiological changes. Extrapolating the data, the development of iNPH typical changes might be an insidious process and the development of tight high convexity might be a variable and nonlinear process. Further studies with larger sample sizes are necessary to put these findings into the pathophysiological perspective of the development of iNPH.

\begin{abstract}
Authors' contributions
DCE was responsible for conception and design, conducted acquisition, analysis and interpretation of data; drafting of manuscript and gave final approval of the version to be published. SDA has been involved in critical revision of the manuscript. MUS has been involved in critical revision of the manuscript. CB made substantial contributions to acquisition and analysis of data; critical revision of the manuscript. All authors read and approved the final manuscript.
\end{abstract}

\section{Author details}

1 Department of Neurosurgery, University Hospital of Tuebingen, Hoppe-Seyler-Strasse 3, 72076 Tuebingen, Germany. ${ }^{2}$ Department of Neuroradiology,
University Hospital of Tuebingen, Hoppe-Seyler-Strasse 3, 72076 Tuebingen, Germany.

\section{Competing interests}

The authors declare that they have no competing interests.

\section{Availability of data and materials}

All data generated or analyzed during this study are included in this published article. Further details are available from the corresponding author on reasonable request.

\section{Ethics approval and consent to participate}

This study was appraised by the local ethics committee of Tübingen without the necessity of informed consent of patients examined in the past according to German Law §15 Abs. 3 LDSG).

\section{Funding}

Author DE was funded by the Tübingen Program for the promotion of Women in Research.

\section{Publisher's Note}

Springer Nature remains neutral with regard to jurisdictional claims in published maps and institutional affiliations.

Received: 10 October 2017 Accepted: 19 January 2018

Published online: 09 February 2018

\section{References}

1. Adams RD, Fisher CM, Hakim S, Ojemann RG, Sweet WH. Symptomatic occult hydrocephalus normal cerebrospinal-fluid pressure. A treatable syndrome. N Engl J Med. 1965;273:117-26.

2. Kuriyama N, Miyajima M, Nakajima M, Kurosawa M, Fukushima W, Watanabe Y, et al. Nationwide hospital-based survey of idiopathic normal pressure hydrocephalus in Japan: epidemiological and clinical characteristics. Brain Behav. 2017:7:e00635.

3. Brean A, Eide PK. Prevalence of probable idiopathic normal pressure hydrocephalus in a Norwegian population. Acta Neurol Scand. 2008;118:48-53.

4. Jaraj D, Rabiei K, Marlow T, Jensen C, Skoog I, Wikkelsö C. Prevalence of idiopathic normal-pressure hydrocephalus. Neurology. 2014;82:1449-54.

5. Iseki C, Takahashi Y, Wada M, Kawanami T, Adachi M, Kato T. Incidence of idiopathic normal pressure hydrocephalus (iNPH): a 10-year follow-up study of a rural community in Japan. J Neurol Sci. 2014;339:108-12.

6. Wilson RK, Williams MA. Evidence that congenital hydrocephalus is a precursor to idiopathic normal pressure hydrocephalus in only a subset of patients. J Neurol Neurosurg Psychiatry. 2007;78:508-11.

7. Bradley WG, Safar FG, Hurtado C, Ord J, Alksne JF. Increased intracranial volume: a clue to the etiology of idiopathic normal-pressure hydrocephalus? Am J Neuroradiol. 2004:25:1479-84.

8. Nakano H, Bandoh K, Miyaoka M, Sato K. Evaluation of hydrocephalic periventricular radiolucency by dynamic computed tomography and xenon-computed tomography. Neurosurgery. 1996;39:758-63.

9. Qvarlander S, Ambarki K, Wåhlin A, Jacobsson J, Birgander R, Malm J, et al. Cerebrospinal fluid and blood flow patterns in idiopathic normal pressure hydrocephalus. Acta Neurol Scand. 2017:135:576-84.

10. Kuriyama N, Tokuda T, Yamada K, Akazawa K, Hosoda M, Sakai K, et al. Flow velocity of the superior sagittal sinus is reduced in patients with idiopathic normal pressure hydrocephalus. J Neuroimaging. 2011;21:365-9.

11. Kuriyama N, Tokuda T, Miyamoto J, Takayasu N, Kondo M, Nakagawa M. Retrograde jugular flow associated with idiopathic normal pressure hydrocephalus. Ann Neurol. 2008;64:217-21.

12. El Sankari S, Fichten A, Gondry-Jouet C, Czosnyka M, Legars D, Deramond $\mathrm{H}$, et al. Correlation between tap test and CSF aqueductal stroke volume in idiopathic normal pressure hydrocephalus. Acta Neurochir Suppl. 2012;113:43-6. 
13. Ringstad G, Emblem KE, Eide PK. Phase-contrast magnetic resonance imaging reveals net retrograde aqueductal flow in idiopathic normal pressure hydrocephalus. J Neurosurg. 2015;1-8.

14. Eklund A, Smielewski P, Chambers I, Alperin N, Malm J, Czosnyka M, et al. Assessment of cerebrospinal fluid outflow resistance. Med Biol Eng Comput. 2007;45:719-35.

15. Qvarlander S, Lundkvist B, Koskinen L-OD, Malm J, Eklund A. Pulsatility in CSF dynamics: pathophysiology of idiopathic normal pressure hydrocephalus. J Neurol Neurosurg Psychiatry. 2013;84:735-41.

16. Wikkelsø C, Hellström P, Klinge PM, Tans JTJ. The European iNPH Multicentre Study on the predictive values of resistance to CSF outflow and the CSF Tap Test in patients with idiopathic normal pressure hydrocephalus. J Neurol Neurosurg Psychiatry. 2013;84:562-8.

17. Kizu O, Yamada K, Nishimura T. Proton chemical shift imaging in normal pressure hydrocephalus. AJNR Am J Neuroradiol. 2001;22:1659-64.

18. Relkin N, Marmarou A, Klinge P, Bergsneider M, Black PM. Diagnosing idiopathic normal-pressure hydrocephalus. Neurosurgery. 2005;57(3 Suppl):S4-16 (discussion ii-v)

19. Alperin N, Oliu CJ, Bagci AM, Lee SH, Kovanlikaya I, Adams D, et al. Lowdose acetazolamide reverses periventricular white matter hyperintensities in iNPH. Neurology. 2014;82:1347-51.

20. Shinoda N, Hirai O, Hori S, Mikami K, Bando T, Shimo D, et al. Utility of MRI-based disproportionately enlarged subarachnoid space hydrocephalus scoring for predicting prognosis after surgery for idiopathic normal pressure hydrocephalus: clinical research. J Neurosurg. 2017;1-7.

21. Pyykkö OT, Lumela M, Rummukainen J, Nerg O, Seppälä TT, Herukka S-K, et al. Cerebrospinal fluid biomarker and brain biopsy findings in idiopathic normal pressure hydrocephalus. PLoS ONE. 2014;9:e91974.

22. Tullberg M, Rosengren L, Blomsterwall E, Karlsson JE, Wikkelsö C. CSF neurofilament and glial fibrillary acidic protein in normal pressure hydrocephalus. Neurology. 1998;50:1122-7.

23. Hakim S, Adams RD. The special clinical problem of symptomatic hydrocephalus with normal cerebrospinal fluid pressure. Observations on cerebrospinal fluid hydrodynamics. J Neurol Sci. 1965;2:307-27.

24. Eide PK, Sorteberg W. Diagnostic intracranial pressure monitoring and surgical management in idiopathic normal pressure hydrocephalus: a 6-Year review of 214 patients. Neurosurgery. 2010;66:80-90.

25. Miyake H, Ohta T, Kajimoto Y, Deguchi J. Diamox ${ }^{\circledR}$ challenge test to decide indications for cerebrospinal fluid shunting in normal pressure hydrocephalus. Acta Neurochir (Wien). 1999;141:1187-93.

26. Tanaka A, Kimura M, Nakayama Y, Yoshinaga S, Tomonaga M. Cerebral blood flow and autoregulation in normal pressure hydrocephalus. Neurosurgery. 1997:40:1165-7.

27. Rekate HL, Brodkey JA, Chizeck HJ, el Sakka W, Ko WH. Ventricular volume regulation: a mathematical model and computer simulation. Pediatr Neurosci. 1988;14:77-84.

28. Bradley WG. Diagnostic tools in hydrocephalus. Neurosurg Clin N Am. 2001;12:661-84 (viii)

29. Bradley WG. Cerebrospinal fluid dynamics and shunt responsiveness in patients with normal-pressure hydrocephalus. Mayo Clin Proc. 2002;77:507-8.

30. Greitz D. Radiological assessment of hydrocephalus: new theories and implications for therapy. Neurosurg Rev. 2004;27:145-65.

31. Greitz D. Cerebrospinal fluid circulation and associated intracranial dynamics. A radiologic investigation using MR imaging and radionuclide cisternography. Acta Radiol Suppl. 1993;386:1-23.

32. Jeppsson A, Zetterberg H, Blennow K, Wikkelsø C. Idiopathic normal-pressure hydrocephalus: pathophysiology and diagnosis by CSF biomarkers. Neurology. 2013;80:1385-92.

33. Cusimano MD, Rewilak D, Stuss DT, Barrera-Martinez JC, Salehi F, Freedman M. Normal-pressure hydrocephalus: is there a genetic predisposition? Can J Neurol Sci. 2011;38:274-81.

34. Huovinen J, Kastinen S, Komulainen S, Oinas M, Avellan C, Frantzen J, et al. Familial idiopathic normal pressure hydrocephalus. J Neurol Sci. 2016;368:11-8.
35. Takahashi Y, Kawanami T, Nagasawa H, Iseki C, Hanyu H, Kato T. Familial normal pressure hydrocephalus (NPH) with an autosomal-dominant inheritance: a novel subgroup of NPH. J Neurol Sci. 2011;308:149-51.

36. Stolze H, Kuhtz-Buschbeck JP, Drücke H, Jöhnk K, Diercks C, Palmié $\mathrm{S}$, et al. Gait analysis in idiopathic normal pressure hydrocephaluswhich parameters respond to the CSF tap test? Clin Neurophysiol. 2000;111:1678-86.

37. Jaraj D, Rabiei K, Marlow T, Jensen C, Skoog I, Wikkelsö C. Prevalence of idiopathic normal-pressure hydrocephalus. Neurology. 2014;82:1449-54.

38. Tolze H, Kuhtz-Buschbeck JP, Drücke H, Jöhnk K, Illert M, Deuschl G. Comparative analysis of the gait disorder of normal pressure hydrocephalus and Parkinson's disease. J Neurol Neurosurg Psychiatry. 2001;70:289-97.

39. Relkin N, Marmarou A, Klinge P, Bergsneider M, Black PM. INPH guidelines, part II: diagnosing idio-pathic normal-pressure hydrocephalus. Neurosurgery. 2005;57(3 SUPPL):S2-17.

40. Evans WA, Davidoff LM, Dyke CG, King GC. An encephalographic ratio for estimating ventricular enlargement and cerebral atrophy. Arch Neurol Psychiatry. 1942:47:931.

41. Narita W, Nishio Y, Baba T, lizuka O, Ishihara T, Matsuda M, et al. Highconvexity tightness predicts the shunt response in idiopathic normal pressure hydrocephalus. Am J Neuroradiol. 2016;37:1831-7.

42. Fazekas F, Chawluk JB, Alavi A. MR signal abnormalities at $1.5 \mathrm{~T}$ in alzheimer's dementia and normal aging. Am J Neuroradiol. 1987;8:421-6.

43. Bradley WG. CSF Flow in the brain in the context of normal pressure hydrocephalus. AJNR Am J Neuroradiol. 2015;36:831-8.

44. Eide PK, Saehle T. Is ventriculomegaly in idiopathic normal pressure hydrocephalus associated with a transmantle gradient in pulsatile intracranial pressure? Acta Neurochir (Wien). 2010;152:989-95.

45. Greitz D. New view on the CSF circulation: CSF is produced and absorbed by brain capillaries. Neuroradiol J. 2010;23:94-5.

46. Burt VL, Whelton P, Roccella EJ, Brown C, Cutler JA, Higgins M, et al. Prevalence of hypertension in the US adult population. Results from the Third National Health and Nutrition Examination Survey, 1988-1991. Hypertension. 1995;25:305-13.

47. Neuhauser HK, Adler C, Rosario AS, Diederichs C, Ellert U. Hypertension prevalence, awareness, treatment and control in Germany 1998 and 2008-11. J Hum Hypertens. 2015; August:1-7.

48. Akai K, Uchigasaki S, Tanaka U, Komatsu A. Normal pressure hydrocephalus, neuropathological study. Acta Pathol Jpn. 1987;37:97-110.

49. Newton H, Pickard JD, Weller RO. Normal pressure hydrocephalus and cerebrovascular disease: findings of postmortem. J Neurol Neurosurg Psychiatry. 1989;52:804

50. Bech RA, Waldemar G, Gjerris F, Klinken L, Juhler M. Shunting effects in patients with idiopathic normal pressure hydrocephalus; correlation with cerebral and leptomeningeal biopsy findings. Acta Neurochir (Wien). 1999;141:633-9.

51. Zarrinkoob L, Ambarki K, Wåhlin A, Birgander R, Carlberg B, Eklund A, et al. Aging alters the dampening of pulsatile blood flow in cerebral arteries. J Cereb Blood Flow Metab. 2016:36:1519-27.

52. Swenson ER. Pharmacology of acute mountain sickness: old drugs and newer thinking. J Appl Physiol. 2016;120:204-15.

53. Czosnyka Z, van den Boogaard F, Czosnyka M, Momjian S, Gelling L, Pickard JD. The relationship between CSF circulation and cerebrovascular pressure-reactivity in normal pressure hydrocephalus. Acta Neurochir Suppl. 2005;95:207-11.

54. Yamada SM, Masahira N, Kawanishi Y, Fujimoto Y, Shimizu K. Preoperative acetazolamide SPECT is useful for predicting outcome of shunt operation in idiopathic normal pressure hydrocephalus patients. Clin Nucl Med. 2013;38:671-6

55. Vakili S, Moran D, Hung A, Elder BD, Jeon L, Fialho H, et al. Timing of surgical treatment for idiopathic normal pressure hydrocephalus: association between treatment delay and reduced short-term benefit. Neurosurg Focus. 2016;41:E2. 